\title{
Effects of Neonatal Gonadal Steroids on Adult CA3 Pyramidal Neuron Dendritic Morphology and Spatial Memory in Rats
}

\author{
Ceylan Isgor, ${ }^{1}$ Dale R. Sengelaub ${ }^{2}$ \\ ${ }^{1}$ Mental Health Research Institute, The University of Michigan, 205 Zina Pitcher Place, Ann Arbor, \\ Michigan 48109-0720 \\ ${ }^{2}$ Department of Psychology, Indiana University, Bloomington, Indiana 47405
}

Received 3 June 2002; accepted 21 October 2002

\begin{abstract}
The hippocampus is implicated in spatial cognition, which is sexually dimorphic and developmentally sensitive to gonadal steroids. Previously we have shown a sex difference in CA3 pyramidal cell layer volume and neuronal soma size that was reversible with neonatal castration in males or prenatal treatment of females with either testosterone propionate (TP) or a nonaromatizable androgen, dihydrotestosterone propionate, but not estradiol benzoate, all of which correlated with adult water maze navigation. The present study further investigates developmental androgen sensitivity of CA3 pyramidal neurons by measuring dendritic morphology and its relation to adult spatial ability. Female rats were injected with TP on postnatal day (P) 3 and P5 or ovariectomized (OVX) on $\mathrm{P} 2$, and male rats were castrated on $P 2$, with or without testosterone replacement $($ Cas $+T)$. Sham surgery controls were also included. Animals were tested on a water maze in adulthood, sacrificed, and CA3 pyramidal neurons were
\end{abstract}

\section{INTRODUCTION}

The hippocampus has been implicated in many learning and memory functions including spatial mapping

\footnotetext{
Correspondence to: C. Isgor (isgor@umich.edu).

Contract grant sponsor: NIH (to D.R.S.); contract grant number: AG09309.

Contract grant sponsor: Grant-in Aid from Indiana University (to C.I.).

(C) 2003 Wiley Periodicals, Inc.
}

DOI 10.1002/neu.10200
Golgi-stained and reconstructed in three dimensions using a computer-interfaced morphometry system. Highandrogen groups (control males, Cas $+\mathrm{T}$, TP females) performed better in spatial navigation and exhibited CA3 neurons with longer dendrites, a larger number of dendritic branches, and volumes of influence compared to low-androgen groups (control females, castrated males, OVX). Collectively, these findings indicate that the critical time period for organizational effects of androgens on the CA3 pyramidal neurons includes both prenatal and postnatal life, during which time androgens regulate developmental events such as somal growth and neuronal differentiation, all of which significantly contribute to establishing the sex difference in adult spatial navigation. ๑ 2003 Wiley Periodicals, Inc. J Neurobiol 55: 179-190, 2003

Keywords: testosterone; estrogen; spatial learning; CA3; dendritic morphology
(O'Keefe and Nadel, 1978), working memory (Olton et al., 1977; Olton and Papas, 1979), place learning (Morris, 1981; Morris et al., 1982), reversal learning (Berger and Orr, 1983), and attentional factors involved in associative conditioning (Mackintosh, 1975). Although the hippocampus may serve several different functions, a collective body of data from maze navigation, electrophysiologic, and lesion studies in rats repeatedly indicates that the hippocampus significantly contributes to the processing of spatial information (Olton and Samuelson, 1976; O'Keefe and Nadel, 1978; Olton et al., 1978; Olton and Papas, 
1979; Morris, 1983; Jarrard et al., 1984). Spatial navigational ability is sexually dimorphic. Male rats not only perform better in maze tasks (Dawson, et al., 1975; Stewart et al., 1975; Joseph, 1979; Einon, 1980; McGee, 1982; Beatty, 1984; Beatty, 1992) but also utilize different navigational strategies compared to female rats (Williams et al., 1990; Williams and Meck, 1991). Sex differences in spatial navigation have been attributed to organizational effects of gonadal hormones. Neonatal castration of male rats and administration of testosterone to female rats during 10 days postpartum were successful in reversing the sex difference in maze navigation (Dawson et al., 1975; Stewart et al., 1975; Joseph et al., 1978; Williams et al., 1990). More recently, a prenatal component has been implicated in the critical period in which gonadal steroids can organize sex differences in spatial ability in rats (Isgor and Sengelaub, 1998).

Perinatal gonadal steroid manipulations can alter hippocampal morphology, which correlates with spatial performance in adulthood. Roof and Havens (1992) have shown that systemic testosterone propionate (TP) injections of females on postnatal day (P)3 and P5 can reverse the sex difference in water maze navigation as well as masculinize the dentate gyrusgranule cell layer width and thickness in adult rats. Prenatal (embryonic day 16 to birth) manipulations of gonadal steroids (i.e., antiandrogen treatment in males, and androgen or estrogen treatments of females) can also reverse sex differences in the CA1 and CA3 pyramidal cell layer volumes and neuronal soma sizes, which correlate with water maze navigation in adult animals (Isgor and Sengelaub, 1998).

The hippocampal pyramidal cell layer contains abundant androgen and estrogen receptors (Vito and Fox, 1982; Clancy et al., 1991; Shughrue and Merchenthaler, 2000; Solum and Handa, 2001) as well as detectable aromatase activity (MacLusky et al., 1987) in embryonic and neonatal rat brain. Hence, it is plausible that both androgens and estrogens can influence the development of the hippocampal pyramidal cell layer perinatally, resulting in differences in adult spatial navigation. Previously we have shown that although systemic prenatal treatment of male rats with an antiandrogen, flutamide, resulted in feminization of the CA1 and CA3 fields in both laminar volume and neuronal soma size, there seems to be an estrogenic component in the masculinization of the CA1 but not the CA3 pyramidal cell layer in females (Isgor and Sengelaub, 1998). Specifically, the CA3 pyramidal cell layer volume and neuronal soma size were found masculinized in females by prenatal TP or a nonaromatizable androgen, dihydrotestosterone propionate (DHTP), but not estradiol benzoate (EB) in- jection, whereas the CA1 pyramidal cell layer volume and neuronal soma size were masculinized by prenatal TP or EB but not DHTP injection. Although organizational effects of estrogen on hippocampal morphology need to be investigated further, in the adult female, an estrogen-dependent regulation of CA1 but not CA3 pyramidal neuron dendritic spine density has also been reported (Gould et al., 1990; Woolley et al., 1990; Woolley and McEwen, 1992), reinforcing the estrogen sensitivity of CA1 but not CA3 pyramidal neurons. Moreover, even though neonatal androgens have been repeatedly linked to better spatial ability and its underlying hippocampal mechanisms, there has been controversy establishing a link between estrogen, spatial ability and hippocampal morphology in both developing and adult animals. Although cyclic changes in CA1 pyramidal neuron dendritic morphology (spines) is robust (Gould et al., 1990; Woolley and McEwen, 1992), endogenous or exogenous ovarian hormone effects on spatial navigational ability both in the developing and mature animals yielded reports of positive, negative, or no correlations (Dawson et al., 1975; Williams et al., 1990; Berry et al., 1997; Warren and Juraska, 1997; Daniel et al., 1999; Chesler and Juraska, 2000).

Most of the emphasis in studies of spatial ability and hippocampal correlates has been on the dentate gyrus-granule cell layer (Roof and Havens, 1992) and the CA1 pyramidal cell layer (McEwen et al., 1997; Chesler and Juraska, 2000; Warren and Juraska, 2000). However, the CA3 pyramidal cell layer is also emerging as a hippocampal region critical in spatial memory. Recently it has been shown that Fos expression in the CA3 region of the hippocampus is obligatory for spatial memory formation (He et al., 2002), and that the CA3 but not the CA1 region or dentate gyrus displays water maze learning-associated changes in gene expression (Zhao et al., 2000). A complex sex difference has also been reported in CA3 pyramidal neuron dendritic morphology (Fitch et al., 1989; Juraska et al., 1989) and mossy fiber-CA3 pyramidal neuron synapses (Madeira et al., 1991). Furthermore, a decline in synaptic density in CA3 neurons in aged male rats has been linked to agerelated deficits in spatial ability (Geinisman et al., 1995), which suggests that circulating androgens may be important in sustaining the CA3 neuron synapses.

Given the androgen-dependent sex difference we observed in the organization of CA3 pyramidal cell layer volume, neuronal soma size and subsequent spatial ability in adult rats earlier, in this study we examined the dendritic structure of CA3 pyramidal neurons after a variety of early developmental hormone manipulations. Although it has been established 
that adult female CA3 pyramidal neuron dendritic spines do not show estrogen-mediated changes (Woolley et al., 1990), the role of neonatal gonadal steroids on CA3 pyramidal neuron dendritic morphology is currently unknown. It is hypothesized that in addition to their role in gross and cellular morphologic alterations in the CA3 field, androgens and perhaps ovarian hormones during early development also organize dendritic morphology of CA3 pyramidal neurons, all of which significantly contribute to sex differences in spatial ability in adult animals. To test this hypothesis, adult spatial navigational ability and CA3 dendritic morphology were assessed after neonatal androgen/estrogen manipulations in the rat.

\section{METHODS}

\section{Developmental Manipulation}

Twelve pregnant, Sprague-Dawley females were obtained from a commercial breeder (Harlan Laboratories, Indianapolis), and litters were used to form six experimental groups: castrated males, sham-castrated males, castrated males with testosterone replacement $(\mathrm{Cas}+\mathrm{T})$, ovariectomized females (OVX), sham-OVX, and TP-injected females $(n=6$ per group). On P2 (day of birth = P1), pups were sexed by measuring anogenital distance, anesthetized briefly by hypothermia, and gonads were removed bilaterally. Sham surgeries were conducted in the same fashion without the removal of gonads. After surgery, pups were warmed on a heating pad and returned to their mothers. Two androgen treatment regimes were employed. The female TP treatment was modeled after the method of Roof and Havens (1992) as well as our previous work (Isgor et al., 1995). However, neither of these studies examined dendritic morphology, and it was possible that the critical period for dendritic development of CA3 neurons could extend beyond the early postnatal period. The dosage and treatment regime used after castration in males are identical to those previously employed in studying androgen-mediated dendritic growth in male rats throughout the first 7 postnatal weeks (Goldstein et al., 1990; Goldstein and Sengelaub, 1993). In those studies, this dose was simply chosen to ensure sufficient androgen exposure; plasma titers were not measured, and leakage after injection, hormone availability, and metabolism would all potentially compromise treatment at a lower dose. Following surgery, Cas $+\mathrm{T}$ males received daily $\mathrm{TP}$ injections $(0.5 \mathrm{~mL}$, containing $0.1 \mathrm{mg} /$ day $)$ until $\mathrm{P} 28$, at which time they were implanted with silastic capsules containing crystalline testosterone $(\mathrm{T} ; 45 \mathrm{~mm}$, a length that results in plasma $\mathrm{T}$ levels typical of young adults. Pups were weaned on P28, and housed in gang cages (six per cage) until 3 months of age, after which they were transferred to individual cages. During the entire experiment, animals were kept on a 12:12 light:dark cycle, and a free-feeding schedule. Throughout the experiment, animals were treated in accordance with National Institute of Health guidelines on laboratory animal use and care.

\section{Behavioral Assessment}

At adulthood (60+ days), spatial navigation was assessed in the Morris water maze. The water maze was $2.5-\mathrm{m}$ in diameter and $45-\mathrm{cm}$ in depth. The maze was filled with water at room temperature $\left(27^{\circ} \mathrm{C}\right)$ to a depth of $40 \mathrm{~cm}$ and the water was made opaque with nondairy creamer. A circular Plexiglas platform $10-\mathrm{cm}$ in diameter was used as the escape platform. The maze was divided into four quadrants, each containing a possible location for the escape platform in the middle of the quadrant. When the maze was filled with water, the escape platform was submerged $1.5-\mathrm{cm}$ under water. A radio was located in one corner of the room for audio extramaze cues, and a $2.5 \times 2.5-\mathrm{m}$ curtain with large geometric patterns was hung on the front wall for visual extramaze cues. The experimenter was in the room during all trials. In addition, behavior was monitored by an overhead video camera linked to a video recorder.

Pretraining consisted of two 2-min trials in which animals were introduced to the maze individually and allowed to swim about the maze freely. In the first trial, the maze was filled but the escape platform was not included. The quadrant in which each animal spent the least amount of time was recorded. The recorded quadrant for each animal was designated as the critical quadrant (i.e., the quadrant in which the escape platform would be located) to control for place bias. In the second pretraining trial, the escape platform was placed in the critical quadrant for each animal. Animals were introduced to the maze from the vicinity of the escape platform, and were manually assisted to climb on the platform.

Following pretraining, latencies to the submerged platform were measured in escape training. Escape latency (i.e., the time elapsed between introduction to the maze and climbing on the escape platform) was recorded to index each animal's spatial navigation ability. On each trial, animals were introduced to the maze from a randomly chosen quadrant, facing the wall of the maze. Extramaze cues were present during all training trials. A trial lasted until either the animal climbed on the escape platform or $2 \mathrm{~min}$ had elapsed. Animals were allowed to stand on the escape platform for $30 \mathrm{~s}$ once they had climbed on it or after $2 \mathrm{~min}$ had elapsed. After each trial, animals were dried and returned to their home cages. Escape training was conducted twice a day (intertrial interval $=4 \mathrm{~min}$ ) for 8 training days, and escape latencies for each day were averaged to obtain trial blocks.

Following the escape navigation, cued escape training was conducted for four trials. A $20-\mathrm{cm}$ high $\times 3$-cm wide PVC pipe painted with black and white horizontal stripes was attached to the escape platform and was used as a visual cue. The location of the cued escape platform was chosen randomly among the three noncritical quadrants for each rat and shifted to a new location on each trial. Latency to the cued escape platform was measured for all trials. 
Statistical analyses of maze behavior consisted of repeated-measures ANOVAs (groups $\times$ trial blocks), a oneway ANOVA for the last trial block, and appropriate planned comparisons as described below. All statistics reported were for two-tailed probability tests.

\section{Histology}

Three weeks after behavioral assessments, animals were overdosed with urethane and then perfused transcardially with $0.9 \%$ saline only. Brains were removed and processed using a modified Golgi stain (Glaser and Van Der Loos, 1981), which allows visualization of whole neurons including processes. Brains remained immersed in Golgi-Cox solution for 21 days, at which time they were dehydrated and embedded in celloidin. Coronal sections through the hippocampus were cut and collected at $200 \mu \mathrm{m}$. Tissue was further processed following the protocol described by Glaser and Van Der Loos (1981), omitting counterstain.

\section{Measures of Dendritic Morphology}

Five animals per group were selected for dendritic analyses. For each animal, 10-12 CA3 pyramidal neurons were reconstructed at a final magnification of $900 \times$, and dendritic morphology was quantified using a three-dimensional computer-interfaced morphometry system (Eutectic, NTS, $N$ $=328$ neurons). To be selected for analysis, Golgi-impregnated pyramidal neurons had to be relatively isolated from neighboring impregnated neurons to allow identification of dendrites that belonged to specific cells, and had to contain dark and consistent impregnation throughout the extent of the neuron. Pyramidal neurons were sampled systematically and meted the selection criteria described below from all sections throughout the anterior/posterior extent of the hippocampal CA3 field. The sample included equal representation of both long- and short-shaft populations as described in Fitch et al. (1989). In addition, roughly half of the sample per each group (25-30 neurons out of a total of 50-60 neurons) belonged to the CA3a, CA3b, and the posterior/ ventral subpopulations, whereas the other half was sampled exclusively from the CA3c subpopulation of the pyramidal cell layer following the description of Turner et al. (1995). This was particularly important for nonbiased and comprehensive sampling because it has been demonstrated that significant differences exist in the dendritic length, branch number, and volume of extent of neurons between the larger and more complex CA3a, CA3b, posterior/ventral neurons, and the generally smaller CA3c neurons (Turner et al., 1995). Lastly, in selection of neurons for reconstruction, effort was made to equally sample from neurons with deep and superficial positioning of the soma within the cell layer. Only CA3 neurons with a typical single apical shaft were included in the analyses, which automatically discarded neurons with unusual morphology at the extremes of the septal/temporal axis. Measurements of dendritic morphology included total dendritic length and total number of dendritic branches per neuron. The volume of influence (the

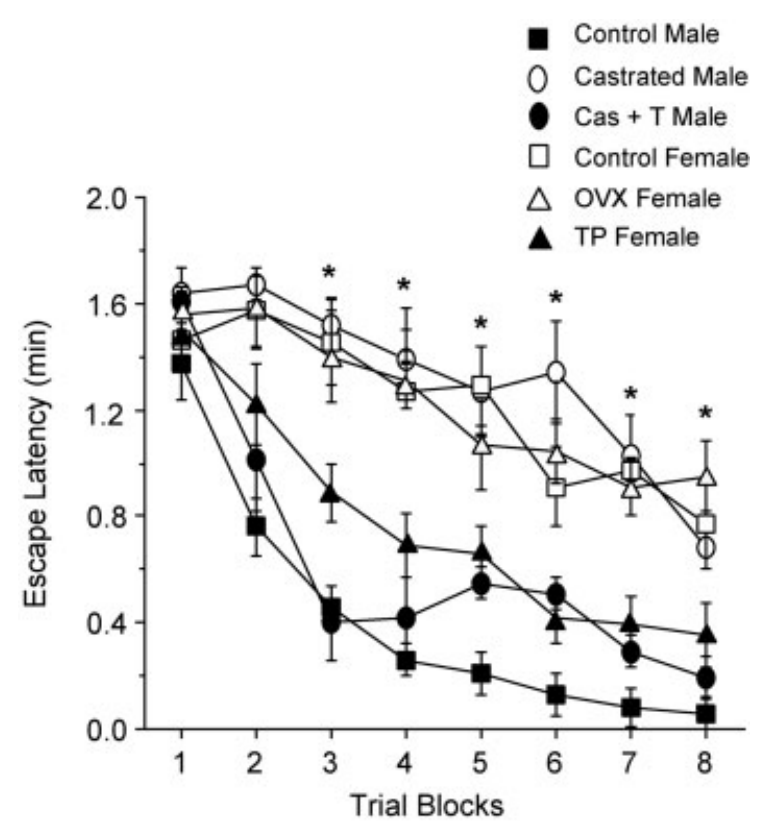

Figure 1 Mean escape latency across trial blocks for control males, castrated males, Cas $+\mathrm{T}$, control females, OVX, and TP females. Points represent means \pm S.E.M.s. High-androgen groups (represented by solid symbols; control males, Cas $+\mathrm{T}$, and TP females) acquired escape latencies quicker than low-androgen groups (represented by open symbols; control females, castrated males and OVX) across trial blocks 3-8. *Indicates $p<.05$.

minimal rectilinear three-dimensional space occupied by the neuron including all of its processes) was also computed for each neuron by the automated morphometry system. Measurements were collected separately for the short- and longshaft CA3 pyramidal neurons and for the apical and basilar dendrites to determine if there were sex and/or steroid effects dependent upon the subpopulation of neurons and type of dendrite. Measurements were corrected for shrinkage of the tissue at the completion of analyses.

Statistical analyses of morphologic data consisted of repeated-measures ANOVAs (group $\times$ type of neuron; group $\times$ type of dendrite) followed by one-way ANOVAs, and planned comparisons as described below. All statistics reported were for two-tailed probability tests.

\section{RESULTS}

\section{Spatial Navigation}

To assess whether there were sex and/or steroid effects in spatial navigation, escape latency to a submerged platform was plotted across trial blocks for control males, castrated males, Cas $+\mathrm{T}$ males, control females, OVX females, and TP females (Fig. 1). A repeated-measures ANOVA revealed a significant dif- 

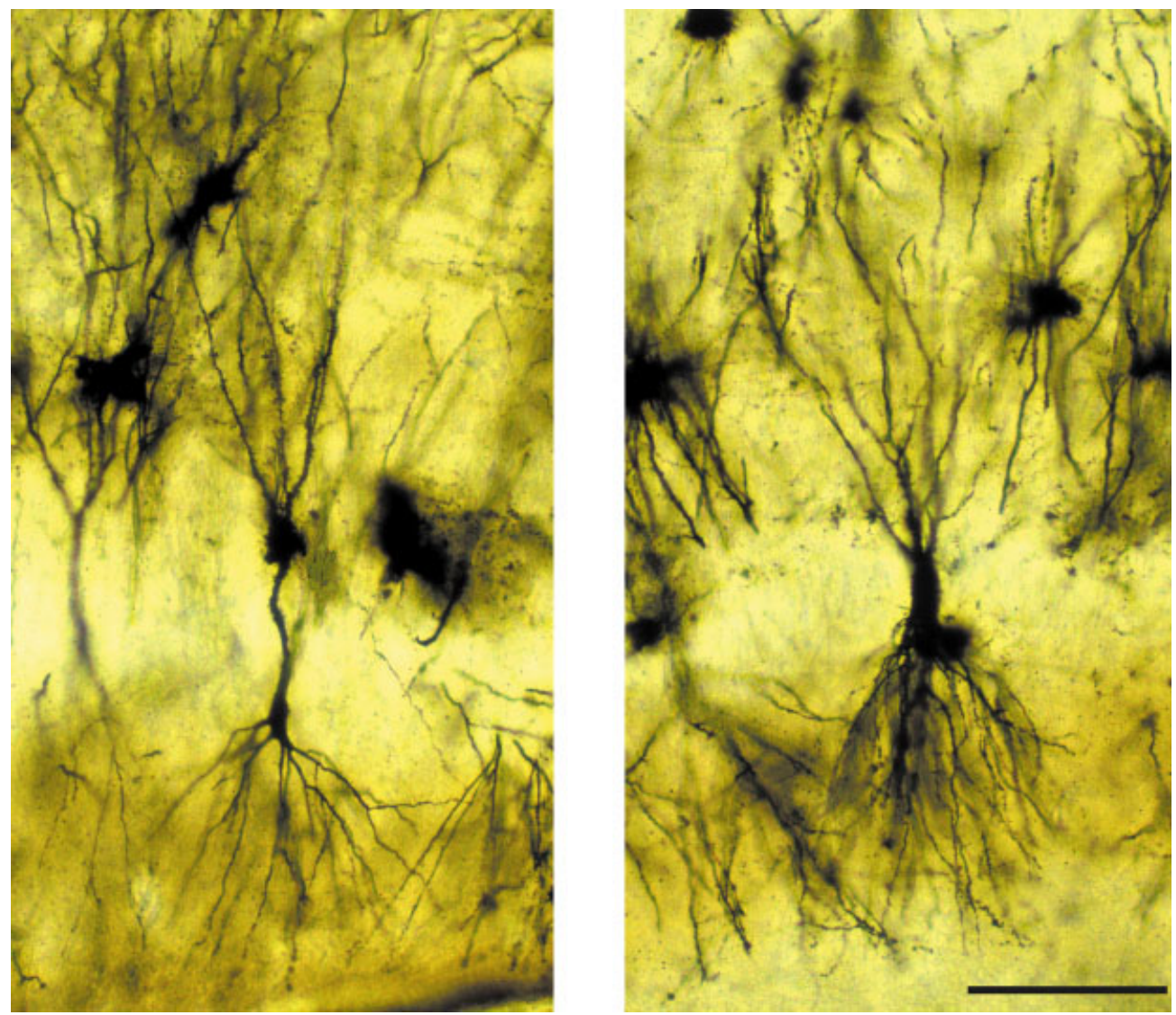

Figure 2 Photomicrographs of Golgi-stained long-shaft CA3 pyramidal neuron from a control female (left) and a short-shaft CA3 pyramidal neuron from a TP-treated female rat (right). Up, dorsal; scale bar, $125 \mu \mathrm{m}$.

ference in the escape latencies between groups across trial blocks, $F(35,210)=4.25, p<.0001$. The main effect of trial blocks was significant, $F(7,210)$ $=31.56, p<.0001$, indicating that on average, all group's performance significantly improved with training. The main effect of groups was also significant, $F(5,210)=102.35, p<.0001$, indicating that groups were significantly different in their escape latencies regardless of trial blocks. Furthermore, oneway ANOVAs conducted in escape latencies on each trial block showed significant differences between groups on blocks $3-8, F \mathrm{~s}(5,30)>16.97, p \mathrm{~s}<.001$. At critical trial blocks, planned comparisons revealed significant sex differences in escape latencies in favor of males $(p \mathrm{~s}<.001)$. TP treatment of females resulted in a significant decrease in escape latency, while castration of males resulted in a significant increase in latencies compared to sham controls at each affected trial block $(p s<.0001)$. Castrated males and OVX females showed latencies that did not differ significantly from those of control females throughout the escape training. Overall, high-androgen groups (con- trol males, Cas $+\mathrm{T}$ males and TP females) found the escape platform more rapidly and had significantly shorter escape latencies at the completion of escape training compared to low-androgen groups (control females, castrated males, and OVX females).

All groups acquired the cued version of the escape training at comparable rates, $F(15,90)=1.63, p$ $>.05$, indicating that the observed differences in the escape training were not due to motor ability or motivational differences between groups.

\section{Measures of Dendritic Morphology}

Complete impregnation of numerous CA3 pyramidal neurons was apparent with the Golgi-Cox method employed (Fig. 2). Reconstructions from representative neurons for control females, TP-treated females, control males, and castrated males are depicted in Figure 3 (A)-(D). Because relatively thick sections were taken through the hippocampus, the arbors of pyramidal neurons selected for analysis were completely contained within a single section. Pyramidal 


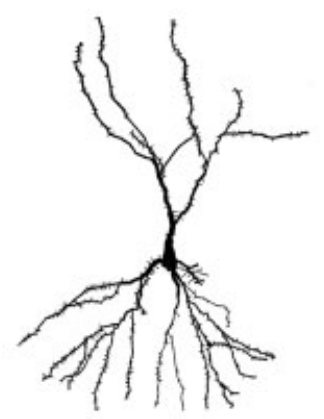

A

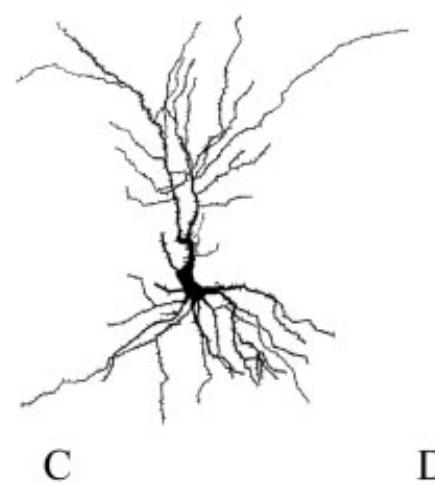

D

Figure 3 Computer-assisted reconstructions of Golgistained CA3 pyramidal neurons from a control female (A), a TP-treated female (B), a control male (C), and a castrated male (D) rat. Scale bar, $60 \mu \mathrm{m}$.

neurons were selected with equal percentage from long- and short-shaft populations (Fig. 2, left, right, respectively; see Fitch et al., 1989, for description) as well as from subpopulations displaying relatively large (i.e., CA3a, CA3b, posterior/ventral) and small (i.e., CA3c) dendritic arbors for each group (see Turner et al., 1995, for description).

Dendritic Length. Total dendritic length for each CA3 pyramidal neuron was computed from threedimensional reconstructions and averaged for control males, castrated males, Cas $+\mathrm{T}$ males, control females, OVX females, and TP females. A repeatedmeasures ANOVA was conducted on dendritic lengths for the apical and basilar dendrites and showed no interaction between groups and the type of dendrite, $F(5,24)=1.98, p>.05$. Because sex and/or steroid treatments in the present study did not differentially alter the apical and basilar dendrites, measurements from both types of dendrites were collapsed for each animal and group [Fig. 4(A)]. Mean CA3 dendritic length was roughly $25 \%$ shorter in control females and $35 \%$ shorter in castrated males compared to that of control males. Moreover, TP females had roughly a $35 \%$ increase in average dendritic length relative to that of control females. A one-way ANOVA performed on dendritic lengths showed a significant difference between groups, $F(5$, $24)=22.66, p<.0001$. Planned comparisons revealed significantly longer dendrites in control males and TP females compared to those of control females, as well as in control males compared to castrated males $(p s<.0001)$. The effects observed did not seem to be specific to short- or long-shaft neurons as indicated by no significant interaction in repeatedmeasures ANOVA between groups and type of neuron, $F(5,24)=1.89, p>.05$ [Fig. 4(B)]. However, sex and steroid effects in dendritic length were larger in the short-shaft population compared to the longshaft population. Females had shorter dendritic lengths in both the short-shaft $(30 \%)$ and long-shaft (20\%) CA3 neurons in comparison to males. Removal of androgens via castration in the male and administration of TP in the female were associated with more pronounced changes in the total dendritic length per neuron in the short-shaft (35-40\%) than in the longshaft (roughly 25\%) populations, even though all were significant $(p s<.001)$. In general, short-shaft neurons had significantly longer dendrites than longshaft neurons $(p<.001)$. Regardless of the type of neuron, TP females showed an average dendritic length comparable to that of control males, while castrated males showed an average dendritic length comparable to that of control females. Although Treplaced castrates had an average dendritic length comparable to that of control males, ovariectomy did not significantly alter dendritic length $(p s>.05)$.

Dendritic Branches. Total number of dendritic branches per CA3 pyramidal neuron was computed from three-dimensional reconstructions and averaged for control males, castrated males, Cas $+\mathrm{T}$ males, control females, OVX females, and TP females for the apical and basilar dendrites. A repeated-measures ANOVA showed no differences in the mean number of branches between groups and the type of dendrite, $F(5,24)=3.03, p>.05$. Because sex and/or steroid treatments did not differentially alter the apical and basilar dendrites, dendritic branch measurements were collapsed across the two types of dendrites for each animal and group [Fig. 4(C)]. Mean number of dendritic branches per CA3 neuron was roughly $25 \%$ smaller in control females and in castrated males compared to that of control males. Moreover, TP females had roughly a $20 \%$ increase in the number of dendritic branches compared to control females. A one-way ANOVA conducted on the overall number of 

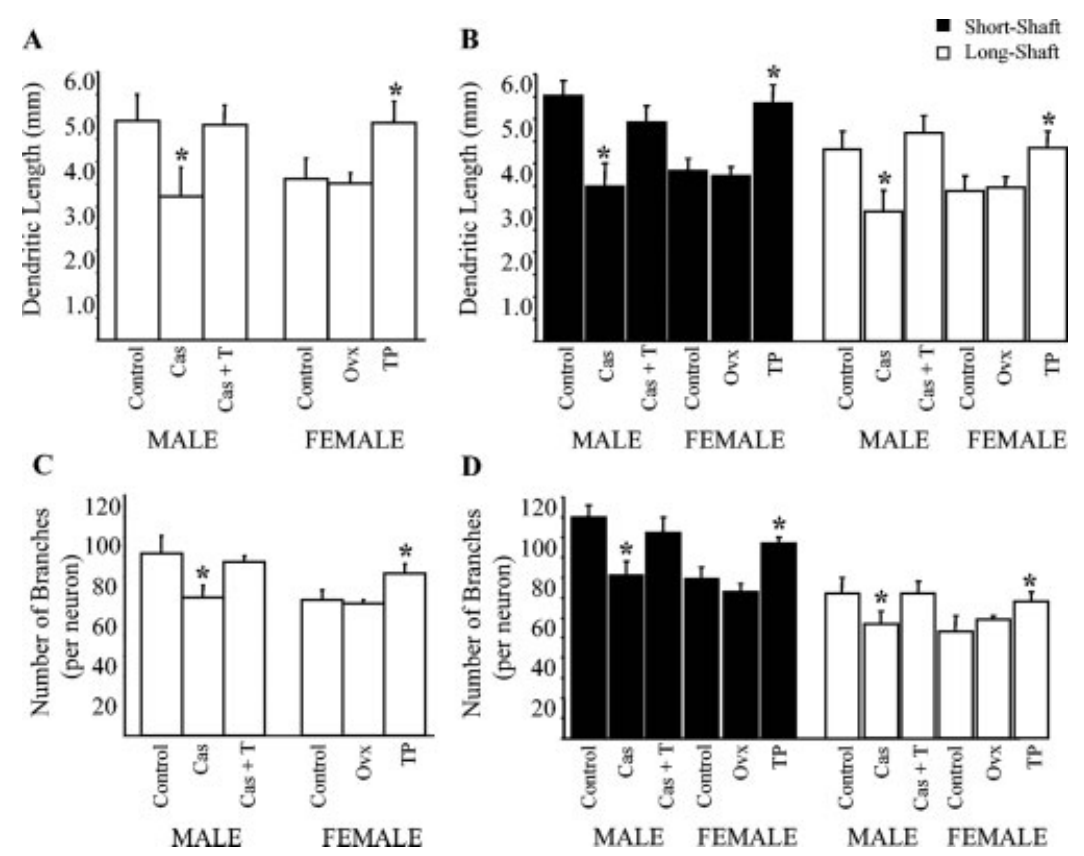

Figure 4 (A). Mean dendritic length of CA3 pyramidal neurons for control males, castrated males, Cas $+\mathrm{T}$, control females, OVX, and TP females. Vertical bars represent means \pm S.E.M.s. Average dendritic length per neuron was significantly higher in high-androgen groups (control males, Cas $+\mathrm{T}$, TP females) compared to low-androgen groups (control females, castrated males, OVX). (B) Mean dendritic length of CA3 neurons in each group divided into short-shaft (solid bars) and long-shaft (open bars) subpopulations. Sex and androgen effects existed in both types of neurons, and at larger magnitudes in the short-shaft population. (C) Mean number of dendritic branches per CA3 pyramidal neuron for control males, castrated males, Cas $+\mathrm{T}$, control females, OVX, and TP females. Vertical bars represent means \pm S.E.M.s. Average branch number per neuron was significantly higher in high-androgen groups (control males, Cas $+\mathrm{T}$, TP females) compared to low-androgen groups (control females, castrated males, OVX). (D) Mean dendritic branch number of CA3 neurons in each group divided into short-shaft (solid bars) and long-shaft (open bars) subpopulations. Sex and androgen effects were found in both types of neurons, and at larger magnitudes in the short-shaft population. *Indicates $p<.05$.

dendritic branches revealed a significant difference between groups, $F(5,24)=20.11, p<.0001$. Planned comparisons showed a significant sex difference in branch number in favor of males $(p<.0001)$. Similar to dendritic length findings, TP females showed a significantly higher number of branches compared to control females, whereas castrated males showed a significantly lower number of branches compared to control males ( $p \mathrm{~s}<.0002$ ), indicating androgen-sensitivity of the sex difference in branch number. A repeated-measures ANOVA showed that the number of dendritic branches per CA3 neuron was not differentially affected by sex or androgen treatments between short- and long-shaft pyramidal neurons, $F(5,24)=2.85, p>.05$ (Fig. 4(D)]. However, the observed sex and steroid effects were slightly larger in the short-shaft population (25-30\%) in comparison to the long-shaft $(20-25 \%)$ population, even though all were significant $(p s<.01)$. In general, short-shaft CA3 neurons contained significantly more dendritic branches than long-shaft neurons $(p<.01)$. Regardless of the type of neuron, the average number of dendritic branches for TP females reached a level comparable to that of control males, whereas the average branch number for castrated males was comparable to that of control females. As in the dendritic length findings, dendritic branch number was also found to be unaltered by ovariectomy, and reached the levels of control males in castrates treated with $\mathrm{T}$ replacement $(p s>.05)$.

Volume of Influence. Volume of influence for each neuron was automatically calculated by the software by connecting the end points of reconstructed dendrites in three dimensions to generate a volume that contain the entire neuron. This measure was used as an indication for neuropil volume and to further ana- 


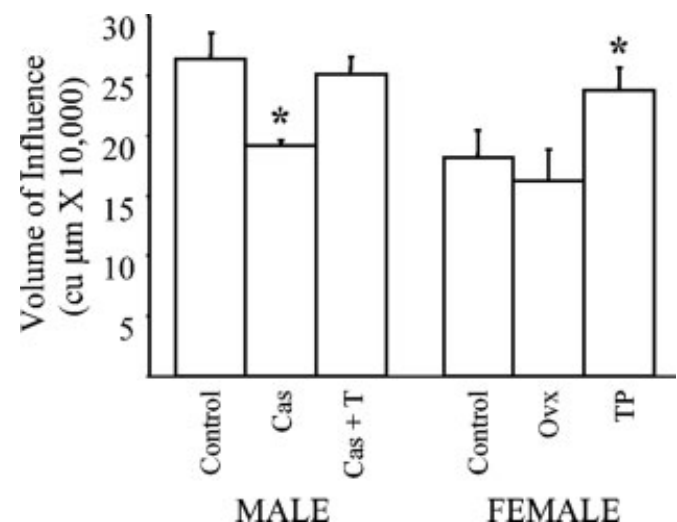

Figure 5 Mean volume of influence of CA3 pyramidal neurons for control males, castrated males, Cas $+\mathrm{T}$, control females, OVX, and TP females. Vertical bars represent means \pm S.E.M.s. Volume of influence was significantly larger in high-androgen groups (control males, Cas + T, TP females) compared to low-androgen groups (control females, castrated males, OVX). *Indicates $p<.05$.

lyze differences in dendritic extent between groups. CA3 pyramidal neuron volume of influence was estimated for each neuron and averaged for control males, castrated males, Cas $+\mathrm{T}$ males, control females, OVX females, and TP females (Fig. 5). Neurons from control females and castrated males had roughly $30 \%$ smaller volumes of influence compared to control males. Moreover, neurons from TP females had roughly a $30 \%$ larger volume of influence compared to that of control females. A one-way ANOVA conducted on the volume of influence between groups showed a significant difference, $F(5,24)=20.45, p$ $<.0001$. Planned comparisons revealed a robust sex difference (control males $>$ control females), which was also significantly altered by androgens (TP females $>$ control females, castrated males $<$ control males; $p$ s $<.0009$ ). The volume of influence for CA3 neurons in TP females was comparable to that of control males, whereas the volume of influence for castrated males was comparable to that of control females. No significant differences were observed in the CA3 volume of influence between OVX females compared to control females, and Cas $+\mathrm{T}$ males compared to control males $(p s>.05)$.

\section{DISCUSSION}

The presence or absence of androgens in the first postnatal week significantly reversed sex differences in spatial navigational ability and CA3 pyramidal neuron dendritic morphology. TP injection of females on P3 and P5 was sufficient to masculinize escape latency to a submerged platform in the water maze navigation task, as well as CA3 pyramidal neuron dendritic length, number of dendritic branches, and volume of influence in adulthood. Ovariectomy during this time did not result in significant changes in either adult spatial navigation or CA3 dendritic morphology. Conversely, castration of males on $\mathrm{P} 2$ successfully feminized water maze navigation and CA3 pyramidal neuron dendritic length, number of dendritic branches, and volume of influence in adulthood, while $\mathrm{T}$ replacement resulted in behavioral and dendritic measures that did not differ from those of intact males. Importantly, both androgen treatment regimes (early postnatal exposure in females and treatment through puberty in males) were sufficient to masculinize both water maze behavior and hippocampal morphology to levels comparable to those of normal males. We conclude that in addition to the previously reported critical period for prenatal androgen effects on CA3 pyramidal neuron somal growth (Isgor and Sengelaub, 1998), there seems to be a postnatal critical period for androgens in organizing CA3 pyramidal neuron dendritic growth, all of which may contribute significantly in producing the sex difference in adult spatial ability.

\section{Neonatal Androgens and Water Maze Navigation}

Behavioral findings of the current experiment are in agreement with previous reports of neonatally androgen-sensitive sex differences in spatial ability (Dawson et al., 1975; Stewart et al., 1975; Joseph et al., 1978; Roof and Havens, 1992). We found that male rats had shorter escape latencies than female rats in finding the submerged platform, and neonatal TP injections of females on P3 and P5 and castration of males on $\mathrm{P} 2$ reversed this sex difference. T replacement of neonatally castrated males resulted in escape latencies comparable to those from intact males. This finding implies that androgens are necessary for producing masculine escape latencies, and that behavioral impairments seen in castrated males are due to lack of androgen. No differences across groups were observed in the cued version of escape acquisition, demonstrating that observed differences in spatial navigational ability were not due to motor or motivational differences.

In the previous neonatal studies it is unclear whether androgen administration or castration effects on spatial navigation involved a direct $\mathrm{T}$ effect or indirect effects of its aromatizable (estradiol) and/or nonaromatizable (dihydrotestosterone) metabolites. Although we did not particularly aim to answer this question in the present 
study, we showed that ovariectomy on P2 did not result in significant changes in escape navigation in females. Therefore, the absence of neonatal ovarian hormones does not seem to critically alter the sex difference in spatial ability in females in the current water maze paradigm. In the male rat, however, neonatal aromatase inhibitor implants have been previously shown to result in deficits in spatial performance (Williams et al., 1990), suggesting a role for local estrogen biosynthesis in the brain for sex differences in spatial navigation. Conversely, it has also been shown that neonatal estradiol treatment is detrimental to maze performance in intact and castrated males (Dawson et al., 1975), demonstrating that the presence of estrogen in early development not only fails to facilitate performance, but also may cause deficits in navigational ability. Moreover, we have previously demonstrated that prenatal EB treatment during the last week of gestation does not change adult spatial ability in female rats, while TP and DHTP treatments significantly masculinize escape navigation (Isgor and Sengelaub, 1998), indicating that developmental estrogen alone, at least in the last week of gestation, is not critical for adult spatial navigation. In the adult literature, spatial ability, specifically performance on tasks involving reference memory, was reported not to differ across the estrous cycle (Berry et al., 1997), to improve with the presence of ovarian hormones compared to the performance of ovariectomized animals (Daniel et al., 1999), as well as to deteriorate with acute estrogen and progesterone replacement after ovariectomy (Chesler and Juraska, 2000). These reports were conroversial in the face of the finding that ovarian hormones either endogenously or exogenously can enhance spine density in the hippocampal granule and pyramidal cells in adult rats (Woolley, 1998; Miranda et al., 1999). More recently, it has been shown that the same estrogen treatments that increased hippocampal dendritic spine density in the adult rat also improved spatial working memory (Daniel and Dohanich, 2001; Sandstrom and Williams, 2001). Although plenty of data exist pertaining to activational effects of gonadal steroids on sexually dimorphic spatial ability, more systematic studies are needed from perinatally androgenized or estrogenized males and females to determine if/how ovarian hormones and local estrogen biosynthesis are involved in organizing this sex difference.

\section{Neonatal Androgens and CA3 Dendritic Morphology}

CA3 pyramidal neuron dendritic length revealed a sex difference in favor of males, which was fully reversed by TP administration in females and castration of males neonatally. $\mathrm{T}$ replacement after neonatal cas- tration produced dendritic lengths comparable to those of intact males. Similar to dendritic length, dendritic branch number per CA3 neuron also showed a sex difference (male $>$ female), which was neonatally androgen-sensitive (TP female $>$ female; castrated male $<$ male), and was maintained by $\mathrm{T}$ replacement in castrated males. Effects reported here were not specific to short- or long-shaft neurons or restricted to only the apical part of the dendritic tree. Differences in dendritic geometry including branch patterns, distribution of processes, and overall shape of dendritic arbors are known to reflect important functional differences (Scheibel and Scheibel, 1970). It is plausible to argue that increased amounts of CA3 pyramidal neuron dendritic material in males and TP females compared to females and castrated males imply that CA3 pyramidal neurons from high androgen groups possess a higher processing capacity compared to those from low-androgen groups, which may underlie the enhanced spatial performance in high androgen groups in the current experiment.

Ovariectomy in our developmental paradigm did not produce significant changes in the $\mathrm{CA} 3$ pyramidal neuron dendritic morphology. However, this finding does not exclude the possibility that exogenously administered androgens may be locally aromatized into estrogen in the hippocampus, and subsequently androgens and estrogen are synergistically involved in sustaining hippocampal dendritic growth. The scope of the current experiment is not suited to completely tease apart differential steroid effects. However, we reported previously that prenatal estrogen could alter the gross and cellular morphology of the CA1 pyramidal cell layer, while only androgens could regulate the CA3 pyramidal neuron morphology (Isgor and Sengelaub, 1998). In adult rats, CA1 but not CA3 pyramidal neuron dendritic morphology was affected by endogenous and exogenous estrogen and progesterone (Woolley et al., 1990; Woolley and McEwen, 1993), further supporting the view that CA3 pyramidal neuron dendritic arbors may not be sensitive to ovarian hormones. An appealing theory is that developmental androgens and estrogens may be acting on separate hippocampal sites (i.e., the CA3 and CA1 fields), and interact in shaping sex differences in overall hippocampal morphology and its behavioral correlates in adulthood. Perinatal transient elevation of estrogen receptors in hippocampal tissue (MacLusky et al., 1979; O'Keefe and Handa, 1990) may define the critical time period when estrogen can exert its maximal effect in masculinizing hippocampal target areas.

Golgi-impregnated CA3 neurons in this study (1) have been sampled equally from short- and long-shaft 
populations as described by Fitch et al. (1989); (2) were selected equally from subpopulations with larger and more complex dendritic arbors such as the CA3a, $\mathrm{CA} 3 \mathrm{~b}$, and posterior/ventral neurons and from smaller neurons with shorter dendrites such as the $\mathrm{CA} 3 \mathrm{c}$ neurons (Turner et al., 1995); and (3) were counterbalanced with regards to location of their somata within the pyramidal cell layer (superficial to deep layers). Sampling criteria of the current study is somewhat different from the only other study where sex differences are reported in the $\mathrm{CA} 3$ pyramidal neuron dendritic field (Juraska et al., 1989). Juraska et al. (1989) reported that in the short-shaft neurons alone and restricted to the apical tree proximal to the soma, females had more dendritic material than males, whereas in the apical tree distal to the soma, males had more dendritic material than females. Even though the short- and long-shaft neurons from both studies were selected using the same criteria as described in Fitch et al. (1989), 85\% of the CA3 pyramidal neurons in the Juraska et al. study were sampled from the CA3b subpopulation, whereas roughly $15 \%$ came from this subpopulation in the current study. In addition to sampling differences, there are also major methodologic differences between the two studies. The current study used computerized three-dimensional reconstructions and corrected for shrinkage to estimate dendritic measurements, whereas Juraska et al. compressed the dendritic field into two dimensions, which can increase variability in measurements and may yield false negatives. Moreover, Juraska et al. used Long-Evans hooded rats as subjects, whereas the current study utilized Sprague-Dawley rats. It is unknown if there are strain differences in dendritic arbors of hippocampal neurons which are sex- and/or steroid-dependent. Furthermore, Juraska et al. sacrificed their subjects at 55 days, whereas in the current study subjects were sacrificed at 90 days. It is becoming evident that the rat hippocampal pyramidal cell layer grows even during normal adulthood in a neuron number independent fashion (Sousa et al., 1998), which suggests that dendritic arbors grow as a part of normal maturation across adulthood, and sex differences may therefore not be fully expressed until young adulthood. Even though we did not detect differences between the apical and the basilar dendritic fields in length and branch number measurements, we did observe that significant effects were larger in the short-shaft population in comparison to the long-shaft population.

Changes in dendritic morphology in a given population of neurons affect its afferents and efferents. As in most subdivisions within the hippocampus, the CA3 pyramidal cell field receives input and sends output in a laminated fashion. For example, the lacunosum moleculare layer of the CA3 field receives laminated afferent input from the enthorhinal cortex, whereas the lucidum layer receives input from dentate granule cells via mossy fibers. The remainder of the dendritic surface of the CA3 pyramidal neuron dendritic tree is primarily taken up with commissural and associational fibers. Dendritic analyses of the CA3 pyramidal neurons in the current study did not focus on a specific portion of the dendritic tree, but rather included three-dimensional analyses of the whole dendritic tree. Although we did not observe gross differences between the apical and basilar dendrites in any dendritic measurement, it is unclear whether effects are homogenously distributed along the dendritic tree or concentrated at certain areas.

Neurons with higher numbers of dendritic branches will also be likely to have longer dendrites. To assess whether differences in the dendritic length between groups were solely determined by differences in the number of dendritic branches, volume of influence was also measured. If the volume of influence is the same between groups while the total dendritic length fluctuates, then the change in dendritic length may be explained by parallel changes in branch number. The current data show that the volume of influence is altered by sex and neonatal androgens in the same direction as dendritic length and branch number. Therefore, it is concluded that dendrites not only have more branches in males and TP females, but they extend longer distances compared to females and castrated males, all of which factor in the increase in dendritic length reported here.

Collectively, our findings indicate that the critical period for organizational effects of androgens on the CA3 pyramidal cell field extends from the last week of gestation (Isgor and Sengelaub, 1998) through the early postnatal period, during which time androgens regulate developmental events such as somal growth and neuronal differentiation, all of which significantly contribute to establishing the sex difference in adult spatial navigation.

We thank Dr. Cara Wellman for her comments on the manuscript.

\section{REFERENCES}

Beatty WW. 1984. Hormonal organization of sex differences in play fighting and spatial behavior. Prog Brain Res 61:320-324.

Beatty WW. 1992. Gonadal hormones and sex differences in nonreproductive behaviors. In: Gerall AA, Moltz H, 
Ward IL, editors. Handbook of behavioral neurobiology, vol 11: sexual differentiation. New York: Plenum, $p$ 85-128.

Berger TW, Orr WB. 1983. Hippocampectomy selectively disrupts discrimination reversal learning of the rabbit nictitating membrane. Behav Brain Res 8:49-68.

Berry B, McMahan R, Gallagher M. 1997. Spatial learning and memory at defined points of the estrous cycle: effects on performance of a hippocampal-dependent task. Behav Neurosci 111:267-274.

Chesler EJ, Juraska JM. 2000. Acute administration of estrogen and progesterone impairs the acquisition of the spatial Morris water maze in ovariectomized rats. Horm Behav 38:234-242.

Clancy AN, Bonsall RW, Michael RP. 1991. Immunohistochemical labeling of androgen receptors in the brain of rat and monkey. Life Sci 50:409-417.

Daniel JM, Dohanich GP. 2001. Acetylcholine mediates the estrogen-induced increase in NMDA receptor binding in CA1 of the hippocampus and the associated improvement in working memory. J Neurosci 21:6949-6956.

Daniel JM, Roberts SL, Dohanich GP. 1999. Effects of ovarian hormones and environment on radial maze and water maze performance. Physiol Behav 66:11-20.

Dawson J, Cheung Y, Lau R. 1975. Developmental effects of neonatal sex hormones on spatial and activity skills in the white rat. Biol Psychol 3:213-229.

Einon D. 1980. Spatial memory and response strategies in rats: age, sex and rearing differences in performance. Q J Exp Psychol 32:473-489.

Fitch JM, Juraska JM, Washington LW. 1989. The dendritic morphology of pyramidal neurons in the rat hippocampal CA3 area. I. Cell types. Brain Res 479:105-114.

Geinisman Y, deToledo-Morrell F, Heller R. 1995. Hippocampal markers of age-related memory dysfunction: behavioral, electrophysiological and morphological perspectives. Prog Neurobiol 45:223-252.

Glaser EM, van der Loos H. 1981. Analysis of thick brain sections by obverse-reverse computer microscopy: an application of a new high-clarity Golgi-Nissl stain. J Neurosci Methods 4:117-125.

Goldstein L, Kurz EM, Sengelaub DR. 1990. Androgen regulation of dendritic growth and retraction in the development of a sexually dimorphic spinal nucleus. J Neurosci 10:935-946.

Goldstein L, Sengelaub DR. 1993. Motoneuron morphology in the dorsolateral nucleus (DLN) of the rat spinal cord: normal development and androgen regulation. J Comp Neurol 338:588-600.

Gould E, Woolley CS, Frankfurt M, McEwen BS. 1990. Gonadal steroids regulate dendritic spine density in hippocampal pyramidal cells in adulthood. J Neurosci 10: 1286-1291.

He J, Kiyofumi Y, Toshitaka N. 2002. A role of Fos expression in the CA3 region of the hippocampus in spatial memory formation in rats. Neuropsychopharmacology 26:259-268.

Isgor C, Sengelaub DR. 1998. Prenatal gonadal steroids affect adult spatial behavior, CA1 and CA3 pyramidal cell morphology in rats. Horm Behav 34:183-198.

Isgor C, Waldroup L, Sengelaub DR, Timberlake W, Schroeder DM. 1995. Neural and hormonal bases of spatial memory in rats. Soc Neurosci Abstr 21:40.

Jarrard LE, Okaichi H, Goldschmidt R, Stewart O. 1984. On the role of hippocampal connection in the performance of place and cue tasks: comparisons with damage to hippocampus. Behav Neurosci 98:946-954.

Joseph R. 1979. Effects of rearing and sex on maze learning and competitive exploration in rats. J Psychol 101:37-43.

Joseph R, Hess S, Birecree E. 1978. Effects of hormone manipulations and exploration on sex differences in maze learning. Behav Biol 24:364-377.

Juraska JM, Fitch JM, Washburne DL. 1989. The dendritic morphology of pyramidal neurons in the rat hippocampal-CA3 area. II Effects of gender and the environment. Brain Res 479:115-119.

MacKintosh NJ. 1975. A theory of selective attention: variation in the associability of stimuli with reinforcement. Psychol Rev 82:276-298.

MacLusky NJ, Clark AS, Naftolin F, Goldman-Rakic PS. 1987. Estrogen formation in the mammalian brain: possible role of aromatase in sexual differentiation of the hippocampus and neocortex. Steroids 50:108-112.

MacLusky NJ, Lieberburg I, McEwen BS. 1979. The development of estrogen receptor systems in the rat brain: perinatal development. Brain Res 178:129-142.

Madeira MD, Sousa N, Paula-Barbosa MM. 1991. Sexual dimorphism in the mossy fiber synapses of the rat hippocampus. Exp Brain Res 87:537-545.

McEwen BS, Alves SE, Bulloch K, Weiland NG. 1997. Ovarian steroids and the brain: implications for cognition and aging. Neurology 48:S8-S15.

McGee MG. 1982. Spatial abilities: the influence of genetic factors. In Potegal M, editor. Spatial abilities: developmental and physiological foundations. New York: Academic Press, p 199-222.

Miranda P, Williams CL, Einstein G. 1999. Granule cells in aging rats are sexually dimorphic in their response to estradiol. J Neurosci 19:3316-3325.

Morris RGM. 1981. Spatial localization does not require the presence of local cues. Learn Motiv 12:239-260.

Morris RGM. 1983. An attempt to dissociate spatial mapping and working-memory theories of hippocampal function. In: Siefert W, editor. The neurobiology of the hippocampus. London: Academic Press.

Morris RGM, Garrud P, Rawlins JNP, O'Keefe J. 1982. Place navigation is impaired in rats with hippocampal lesions. Nature 297:681-683.

O’Keefe J, Handa RJ. 1990. Transient elevation of estrogen receptors in the neonatal rat hippocampus. Dev Brain Res 57:119-127.

O'Keefe J, Nadel L. 1978. The hippocampus as a cognitive map. Oxford: Oxford University Press.

Olton DS, Branch M, Best PJ. 1978. Spatial correlates of hippocampal unit activity. Exp Neurol 58:387-409.

Olton DS, Collison C, Wertz MA. 1977. Spatial memory 
and radial arm maze performance in rats. Learn Motiv 8:289-314.

Olton DS, Papas BC. 1979. Spatial memory and hippocampal system function. Neuropsychologia 17:669-681.

Olton DS, Samuelson RJ. 1976. Remembrance of places passed: spatial memory in rats. J Exp Psychol Anim Behav Proc 2:97-116.

Roof RL, Havens MD. 1992. Testosterone improves maze performance and induces development of a male hippocampus in females. Brain Res 572:310-313.

Sandstrom NJ, Williams CL. 2001. Memory retention is modulated by acute estradiol and progesterone replacement. Behav Neurosci 115:384-393.

Scheibel ME, Scheibel AB. 1970. Of pattern and place in dendrites. Int Rev Neurobiol 13:1-26.

Shughrue PJ, Merchenthaler I. 2000. Evidence for novel estrogen binding sites in the rat hippocampus. Neuroscience 99:605-612.

Solum DT, Handa RJ. 2001. Localization of estrogen receptor alpha (ER alpha) in pyramidal neurons of the developing rat hippocampus. Brain Res Dev Brain Res 128:165-175.

Sousa N, Madeira MD, Paula-Barbosa MM. 1998. Effects of corticosterone treatment and rehabilitation on the hippocampal formation of neonatal and adult rats. An unbiased stereological study. Brain Res 794:199-210.

Stewart J, Skvarenina A, Pottier J. 1975. Effects of neonatal androgens on open-field behavior and maze learning in prepubescent and adult rat. Physiol Behav 14:291-295.

Turner DA, Li XG, Pyapali GK, Ylinen A, Buzsaki G. 1995. Morphometric and electrical properties of reconstructed hippocampal CA3 neurons recorded in vivo. J Comp Neurol 356:580-594.

Vito CC, Fox TO. 1982. Androgen and estrogen receptors in embryonic and neonatal rat brain. Dev Brain Res 1:97110.

Warren SG, Juraska JM. 1997. Spatial and nonspatial learning across the rat estrous cycle. Behav Neurosci 111: 259-256.

Warren SG, Juraska JM. 2000. Sex differences and estropausal phase effects on water maze performance in aged rats. Neurobiol Learn Mem 14:229-240.

Williams CL, Barnett AM, Meck WH. 1990. Organizational effects of early gonadal steroid secretions on sexual differentiation in spatial memory. Behav Neurosci 104:8497.

Williams CL, Meck WH. 1991. The organizational effects of gonadal steroids on sexually dimorphic spatial ability. Psychoneuroendocrinology 16:155-176.

Woolley CS. 1998. Estrogen-mediated structural and functional synaptic plasticity in the female rat hippocampus. Horm Behav 34:140-148.

Woolley CS, Gould E, Frankfurt M, McEwen BS. 1990. Naturally-occuring fluctuations in dendritic spine density on adult hippocampal pyramidal neurons. J Neurosci 10:4035-4039.

Woolley CS, McEwen BS. 1992. Estradiol mediates fluctuation in hippocampal synapse density during the estrous cycle in the adult rat. J Neurosci 12:2549-2554.

Woolley CS, McEwen BS. 1993. Roles of estradiol and progesterone in regulation of hippocampal dendritic spine density during the estrous cycle in the rat. J Comp Neurol 336:293-306.

Zhao W, Cavallaro S, Gusev P, Alkon DJ. 2000. Nonreceptor tyrosine protein kinase pp60c-src in spatial learning: synapse-specific changes in its gene expression, tyrosine phosphorylation, and protein-protein interactions. Proc Natl Acad Sci USA 97:8098-8103. 